\title{
AUTOMATIC GENERATION OF DYNAMIC VIRTUAL FENCES AS PART OF BIM-BASED PREVENTION PROGRAM FOR CONSTRUCTION SAFETY
}

\author{
Amin Hammad \\ Concordia University \\ 1515 St-Catherine West, EV 9.215 \\ Montreal, CANADA \\ Shayan Setayeshgar \\ Concordia University \\ 1515 St-Catherine West, EV 9.415 \\ Montreal, CANADA
}

\author{
Cheng Zhang \\ Concordia University \\ 1515 St-Catherine West, EV 7.634 \\ Montreal, CANADA \\ Yoosef Asen \\ Concordia University \\ 1515 St-Catherine West, EV 9.415 \\ Montreal, CANADA
}

\begin{abstract}
The present research aims to investigate a new method for the automatic generation of Dynamic Virtual Fences (DVFs) as part of a BIM-based prevention program for construction safety following the Safety Code of Quebec Provence in Canada. First, the Safety Code is reviewed to identify the information that has spatial aspects that can be represented in BIM. Then, a method is proposed for automatically identifying falling and collision risks and generating DVFs. In this method, workspaces are generated in BIM based on Work Breakdown Structure (WBS) deliverables, the project schedule, the dimensions of equipment, and the geometry of the building. One set of DVFs for collision prevention is generated based on those workspaces. Another set of DVFs is generated where physical barriers are needed for fall prevention. The generated DVFs are used coupled with Real-time Location System (RTLS) tracking of workers and physical fences to check safety requirements and to provide safety warnings.
\end{abstract}

\section{INTRODUCTION}

It is important to identify risks on construction sites so as to eliminate them before accidents occur. Out of the construction workers that suffer fatal injuries, more are involved in falls than any other single cause (Huang and Hinze 2003). Falls account for double the number of deaths caused by electrocution or being buried in trenches (ASP construction 2003). Using guardrails, safety nets, harnesses, etc., is an effective way to prevent the workers from being at risk of falling. Furthermore, workers should be kept away from hazard areas, such as the workspace for heavy construction equipment. Physical fences are not mandatory for those areas; however, workers should be notified that those areas are dangerous and they should keep a safety distance from them. Construction sites are dynamic and on-site situations are changing in terms of permanent and temporary structures. Therefore, information of the construction site should be updated based on the project progress monitoring, and the prevention measures for safety should be used to give more awareness to workers. This information can be reflected using Building Information Modeling (BIM).

BIM is a new approach to design, construction, and facilities management in which a digital representation of the building process is used to facilitate the exchange and interoperability of information in a digital format (Eastman et al. 2011). Virtual 3D models can be built to represent a realistic scene of the 
construction site. Using BIM is changing the manner of designing and constructing buildings in the construction industry. Moreover, time information can be added to 3D BIM models to generate 4D models which contain the schedule. The relationship of space and time can be accurately described in a systematic way in $4 \mathrm{D}$ modeling.

Using BIM as a tool, several approaches have been proposed in research. Analysis can be carried out for solving conflicts on site based on the spatio-temporal information provided by BIM (Zhang et al. 2011). Hammad et al. (2011) have proposed an approach to visualize data from different sources including a BIM model, video monitoring, and an Ultra Wideband (UWB) Real-time Location System (RTLS). The benefits of the data fusion from different sources can be expected to the improve quality and productivity of the project. Other research has been aiming to improve efficiency, to reduce the cost, and to improve the safety on construction sites. For example, BIM was used as a 4D safety planning tool (Kiviniemi et al. 2011). That research has indicated that BIM technology could present a new way to solve site safety problems. 4D BIM can be a central technology for planning activities related to construction site safety. By displaying the information in the office to the site staff, general understanding of the risks onsite can be improved (Kiviniemi et al. 2011). However, the virtual safety barriers generated in that researchare static and for visualization purpose only. There is no real-time control for safety by using those virtual barriers.

The present research aims to investigate the effective use of BIM both in prevention programs for safety and in real-time safety management. The objectives of the present paper are: (1) to extract the necessary information with spatial aspects from a safety code and to represent this information using BIM; (2) to investigate a method for automatic risk identification, e.g., openings on the floor and floor edges; (3) to define workspaces based on a schedule and reference objects; and (4) to propose a method to generate virtual fences around risk areas for real-time safety management.

\section{PROPOSED APPROACH}

\subsection{Extracting Information with Spatial Aspects from Safety Code}

In the present research, the Quebec Safety Code for the Construction Industry (ASP construction 2003) is reviewed and information that has spatial aspects is extracted either indicating problems or providing solutions, as shown in Table 1.

\subsubsection{Protection Against Falling}

Locations and dimensions of the physical safety barriers that should be installed to prevent falling can be modeled and visualized in the BIM model, for example, a guardrail installed around an opening on the floor and a safety net (Table $1 \mathrm{a}$ and $1 \mathrm{~b}$ ).

\subsubsection{Housekeeping and Means of Access}

The areas for stacking materials or disposal of waste materials should be fenced or identified to prevent injuries caused by falling objects or materials (Table 1c). Places can be identified where the rods for concrete formwork and other protruding parts may have not been cut. Furthermore, BIM can be used to plan the means of access for both permanent and temporary usage, in case of accident. These means of access will make it possible to evacuate workers quickly and will make it easier for first aid to arrive (Table 1d).

\subsubsection{Scaffolding}

BIM can be used to virtually show the places where scaffolding is needed, to identify the edge of the scaffolding and to check if the guardrail is installed (Table 1e and 1f). 


\subsubsection{Confined Spaces}

A confined space is not designed or meant to be occupied by persons, but may on occasion be occupied for doing certain work; can be accessed only by means of a narrow opening; can present health or safety risks for whoever enters because of its design, construction or position; its air quality or inadequate natural or mechanical ventilation; or the materials or substances it contains (Table 1g and $1 \mathrm{~h}$ ). BIM can be used to identify those confined spaces to give workers adequate information as to the risks connected with their work in a confined space and to provide them with the appropriate training, assistance or supervision to allow them to safely perform the work assigned to them.

\subsubsection{Electricity}

Locations of power lines and safety work zones can be identified in BIM (Table 1i and 1j). For example, the minimum approach distance for a power line of 25,000 volts is 3 meters.

\subsubsection{Heavy Machinery and Self-propelled Vehicles}

Workspaces for the equipment can be identified and virtual fences can be generated to protect workers (Table $1 \mathrm{k}$ and 11).

\subsubsection{Trenches and Excavations}

Barriers or barricades at least $0.9 \mathrm{~m}$ high shall be set up around the edge of any trench or excavation whose depth is more than $3 \mathrm{~m}$ (Table $1 \mathrm{~m}$ ). Any sidewalk and any other public way used by pedestrians and skirting a construction site shall be separated by a fence or a wall of at least $1.8 \mathrm{~m}$ if the sidewalk or public way is less than $2 \mathrm{~m}$ away and there is any danger to pedestrians (Table $1 \mathrm{n}$ ).

\subsection{Generating DVFs in BIM}

After the information with spatial aspects is extracted from the Safety Code, two types of safety risks are identified where virtual fences can be generated automatically in the 4D BIM model. The first type is generated where physical guardrails should be installed (e.g., Table 1a, 1c, 1m). The second type is generated for the workspaces that are reserved and from which workers should keep a safe distance (e.g., Table $1 \mathrm{k}, 1 \mathrm{l})$. A method is proposed for automatic risk identification and workspace identification using a 4D BIM model, work breakdown structure deliverables, and associated equipment. Based on the locations where physical barriers should be installed and the workspaces of each equipment, two types of DVFs are generated to protect the workers from safety risks as explained in the following sub-sections. DVFs are defined as temporary objects in the BIM model. The duration of these DVFs is equal to the corresponding tasks' durations. Those DVFs are removed After the task is completed. 
Table 1: Spatial information related to prevention program for safety.

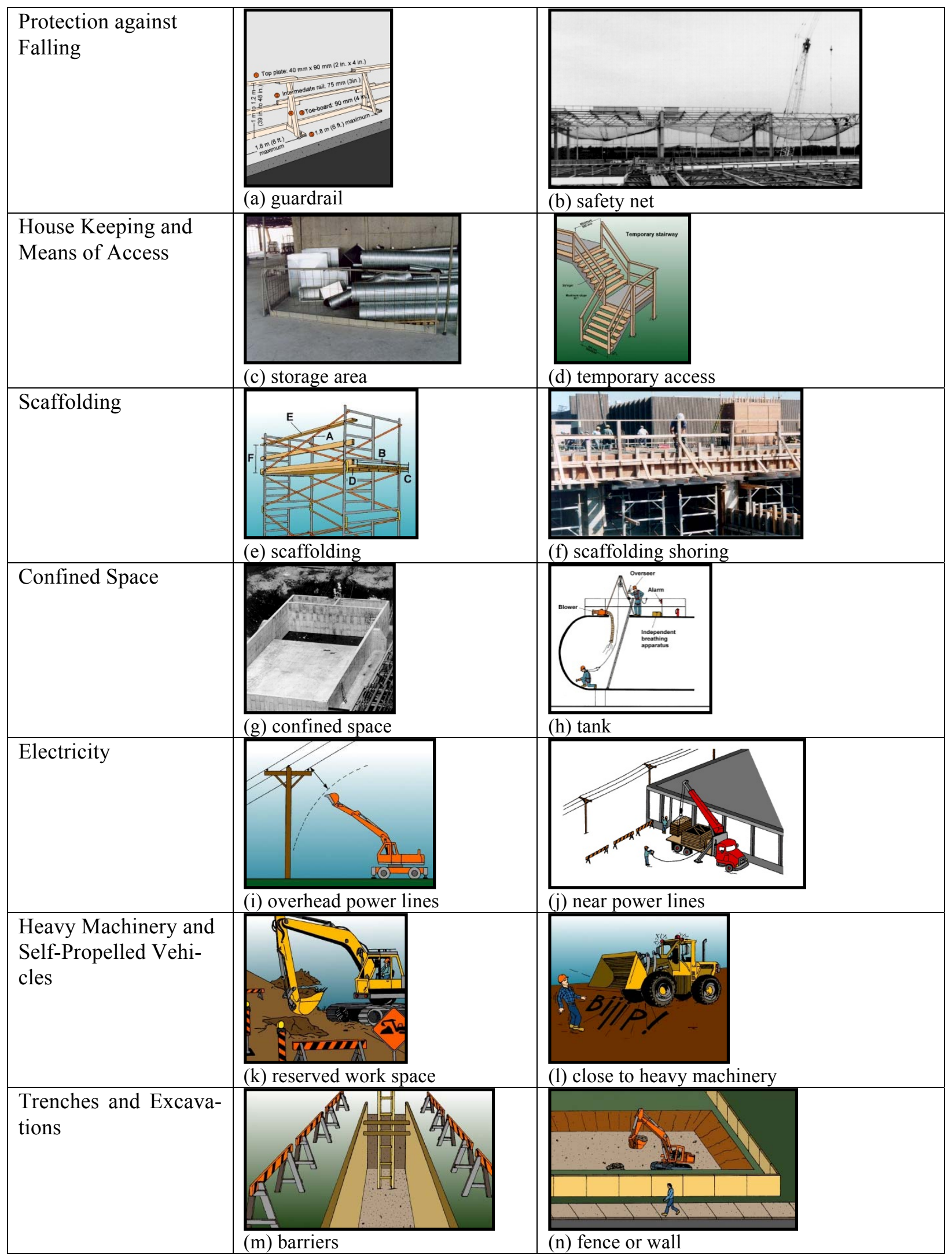




\subsubsection{Fall Prevention DVFs}

Falling is the major risk on site as mentioned in the Introduction; therefore, fall prevention is taken as one of the main purposes for automatic risk searching. Falling happens at unprotected external boundary edges of the slabs (where usually we have external walls) or unprotected openings within the slabs (such as elevators shaft, stairs, etc.). There are three major locations where physical guardrails are mandatory: Building/floor edges, elevators, and stairs.

To establish a prevention program, the first task is to identify potential hazards of construction projects to prepare for safety measures. The proposed method uses a 4D BIM model in order to automatically detect areas with potential risk of falling and the duration of the risk. BIM is used as a visual reference and a database for construction components such as slabs, walls, etc. By determining different construction components and their locations, slabs and related heights from a reference plane can be determined, as well as the edges of the slabs. Slabs' edges are used to find spots with potential risk of falling. Falling happens at an edge where there is a difference between levels of planes located on the two sides of the edge and this is used as the main concept of the automatic detection of areas with potential hazard of falling. Figure 1 shows the relationships at the edges between different adjacent slabs. Figure 2 shows the height relationships at the edges with fall hazards.

Figure 3 shows the flowchart for the automatic detection of areas with fall hazards and the generation of DVFs for these areas. A 4D model is used as a reference to determine slabs and their edges. Upon the detection of an edge $E$ of a slab $S$, the system will check if there is a barrier at this edge or another slab $S$, at the same or lower levels (Figure 1). If another slab is detected, the vertical distance $d$ between the two slabs $\left(S\right.$ and $S^{\prime}$ ) is calculated. The edge is considered as hazardous if: (1) $d$ is more than the specified value in the safety code (e.g. $3 \mathrm{~m}$ according to Quebec Safety Code), (Figure 2-b), or (2) no slab is detected on the other side of $E$ (Figure 2-a \& c).

After defining hazardous edges, DVFs are generated along these edges to protect workers from falling. The heights of the DVFs are determined by the height required for the guardrails, which should be 1 to $1.2 \mathrm{~m}$ above the surface on which the worker is working according to Quebec Safety Code, s. 3.8.3.-1 (ASP Construction, 2003). Given that fences are temporary structures, they should be removed when the hazard of falling no longer exists. Therefore, a task for fencing is added to the project schedule so that it can be visualized in the 4D model as a temporary structure. Information about walls and other physical barriers at a specific edge is required in order to determine the appropriate time for removing the fences. The lifespan of fall hazard of an edge is from the finishing date of the slab to the finishing date of the hazard-eliminating barrier (wall, stair or installation of elevator(s)).

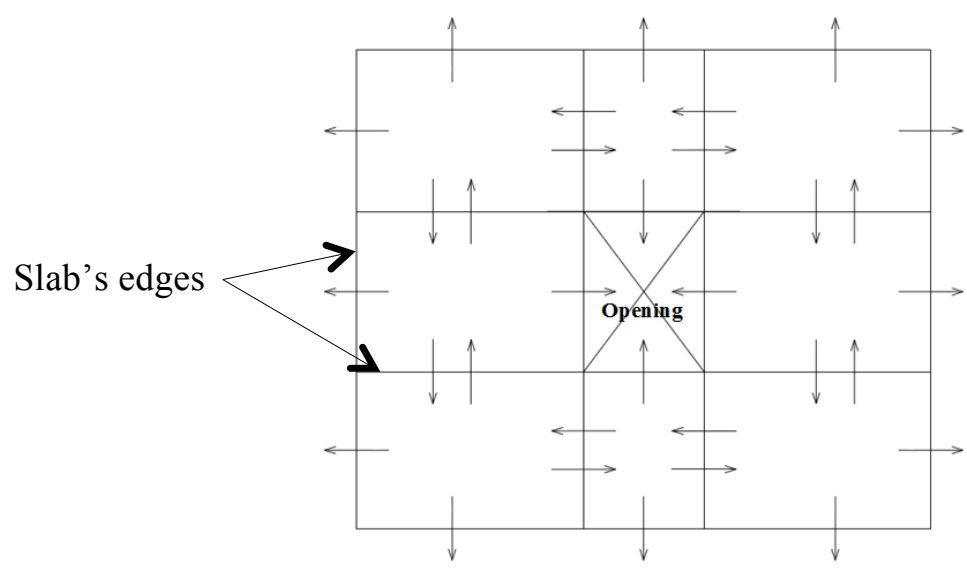

Figure 1: Edge examination for detection of hazardous areas 


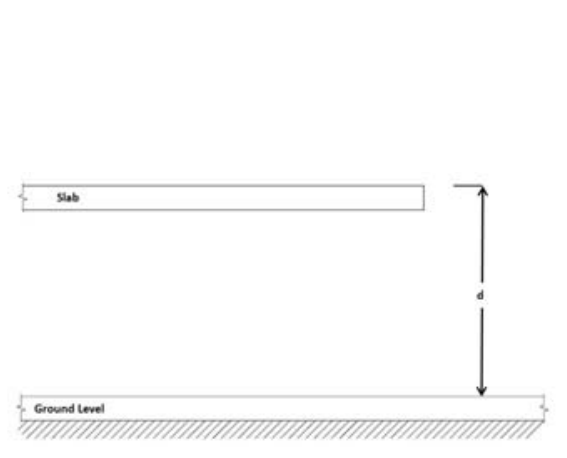

(a) No slab on the other side

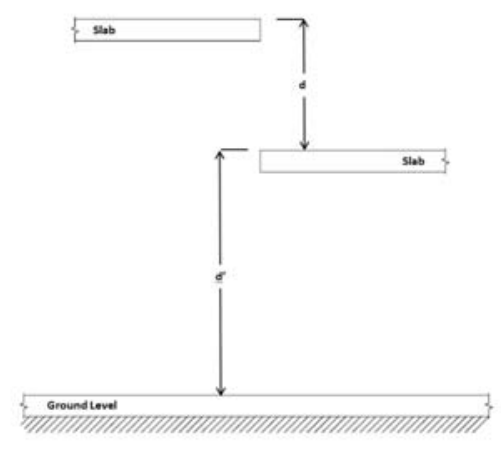

(b) Different levels of slabs

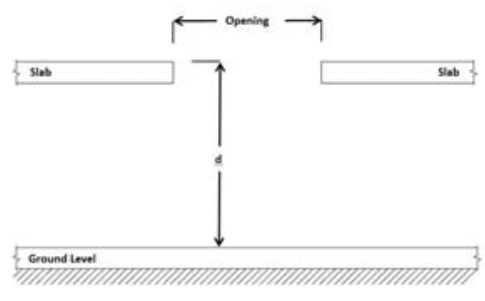

(c) Slab with an opening

Figure 2: Edges with fall hazard

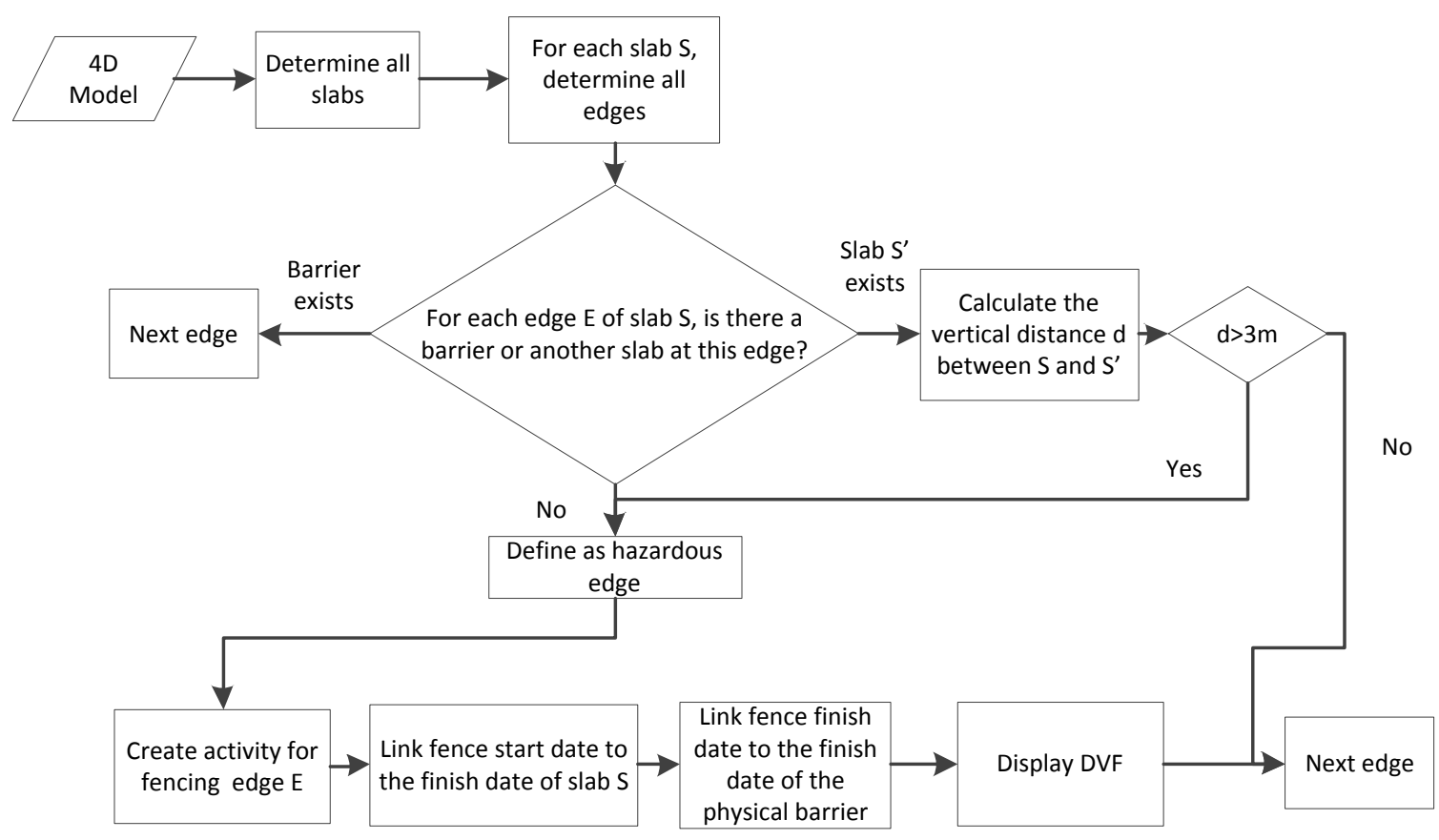

Figure 3: Flowchart of the automatic generation of DVFs in hazardous areas

\subsubsection{Collision Prevention DVFs}

For the collision prevention DVFs, workspace identification is applied for each construction task. Work breakdown structure (WBS) is used to create the hierarchy of the tasks and subtasks of the construction project. Each WBS deliverable is associated with a reference object, which can be building component including Mechanical, Electrical and Plumbing (MEP) components, and temporary structures. Construction pieces of equipment for specific tasks/subtasks are also identified within the WBS. For example, for installing HVAC ducts in a room, the sections of ducts are identified as reference objects. The length, height from the floor, and the location of a section is retrieved from the BIM model. The installation is further divided into subtasks, such as fixing the studs to the ceiling for hanging the ducts. In this case, a scissor lift may be used to help the worker reach the ceiling. Thus, the workspace of the ducts installation task is defined based on the reference object (section of ducts), and dimension of the scissor lift, for that specific task duration. The height of the workspace is equal to the maximum reaching height of the work- 
er, which is the height of the room. A buffer is added around these dimensions to create a box-shaped workspace for the subtask. After the workspace is identified, DVFs are generated around the workspace following the dimensions of the workspace.

\subsection{Real-time Safety Management}

On the construction site, an RTLS is installed to monitor and update the locations of workers and objects on site. Tags are attached to physical barriers and workers to collect location data in real time. The fall prevention DVFs are linked with the physical barriers to check if the physical barriers are installed properly at the right location and with right dimensions for the right duration. The collision prevention DVFs are linked with the locations of workers on site. Warnings are given to the workers if they come too close to the danger areas. Details of real-time safety management can be found in Zhang et al. (2012). Figure 4 Shows the flowchart of the overall proposed approach.

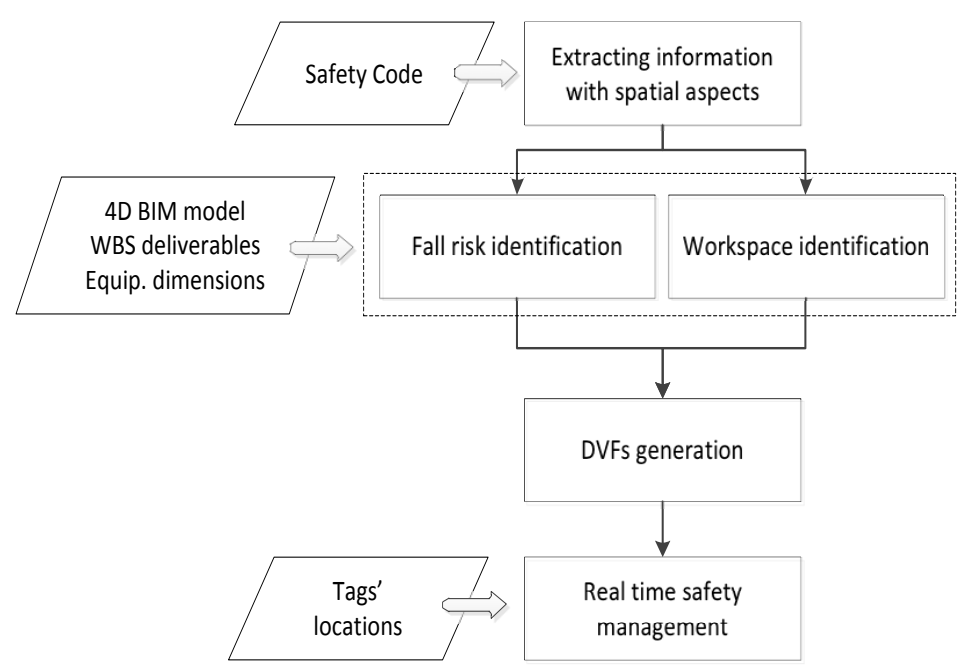

Figure 4: Proposed approach

\section{CASE STUDIES}

Two preliminary case studies are applied to investigate the feasibility of the proposed method. Revit 4D model of the target area is created based on a 2D model using Autodesk Revit Architecture (2012), MEP (2012) and Navisworks (2012). The 4D model has a sufficient level of detail and is used to provide a spatio-temporal reference as well as to visualize different phases of the work, e.g., to show the ducts that have been already installed and those that will be installed later. The 4D model is also used to show the locations of the virtual fences. A real-time location system is installed on site to collect location data of physical guardrails and workers. Using scripts in Navisworks, hot spots are defined around the virtual fences and workers. Once a worker is close enough to the virtual fence, an overlap is found between those hot spots; which means it is unsafe for the worker. In that case, a warning will be sent to the worker to remind him/her to keep a safe distance from that area.

\subsection{Fall Prevention}

The first case study is about automatic searching of fall risks. Slab edges and locations of elevators and stairs are identified in the BIM model. Edges of those areas are found and virtual fences are generated around those areas. The duration for each fence is assigned according to the schedule of the tasks. Figures 
$5 \mathrm{a}$ and $5 \mathrm{~b}$ show the physical guardrails installed around the edge of the floor and the virtual fences generated in the BIM model, respectively. The height of the virtual fences follows the required dimension of the guardrail, which is 1.2 meter. Tags are attached to the top and along the length of the guardrail with some intervals, location data of these tags are collected to compare with the virtual fence locations. If there is any mismatching, the virtual fence in the BIM will be highlighted to show that a correction measure needs to be taken for the guardrail.

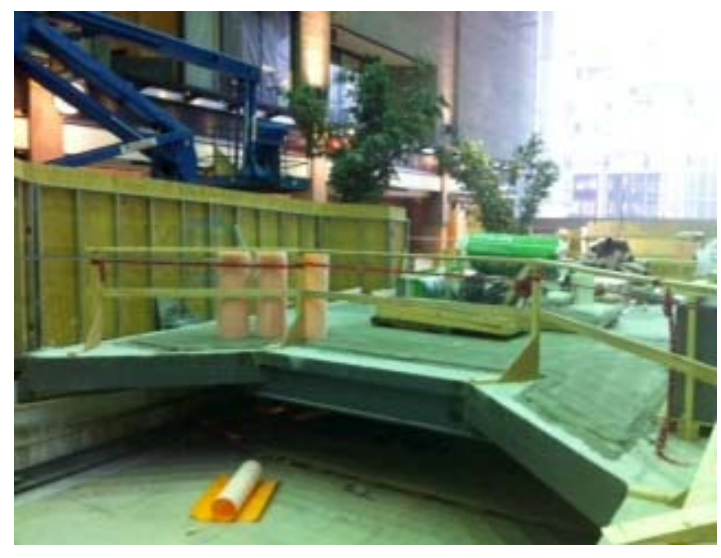

(a) Picture of the site

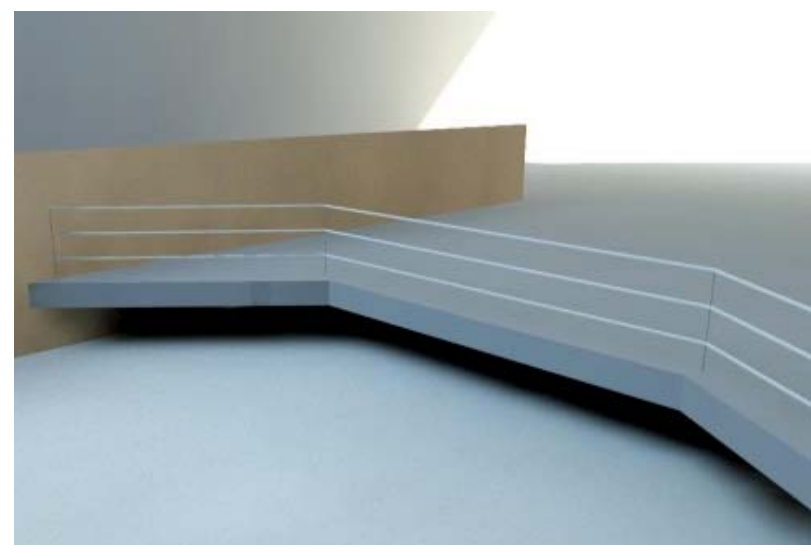

(b) BIM model of the site

Figure 5: Physical guardrails and virtual fences in BIM

\subsection{Collision Prevention}

The second case study is about workspace identification and DVF generation for installing HVAC ducts. Figure 6a shows the HVAC ducts that will be installed in the room and three DVFs for different subtasks in $2 \mathrm{D}$ and $3 \mathrm{D}$.

The three fences are associated with different time durations, as shown in the bottom of Figure 6a. The ducts on the right hand side are scheduled to be installed first, which are highlighted in red color in Figure 6b. A scissor lift (Figure 6c) is used by the worker to reach the ceiling. The workspace of the scissor lift is rectangular and along the window; therefore, the virtual fence is parallel to the workspace to protect workers working in the same room for the time period of the task. Location data of workers and the scissor lift are collected in real-time and their traces are presented in the BIM model providing a spatial reference as shown in Figure 6b.

\section{SUMMARY, CONCLUSIONS AND FUTURE WORK}

Building Information Modeling can be used for visualizing and simulating construction safety measures. Safety related information can be modeled in BIM at each stage of a construction project, while RealTime Location Systems (RTLSs) can be used to monitor workers on site in order to give them safety warnings when necessary based on their relative locations with respect to danger zones specified in the model in order to improve their safety awareness. The present research investigated a new method for the automatic generation of Dynamic Virtual Fences (DVFs) as part of a BIM-based prevention program and real-time safety management for construction safety following the safety code of Quebec Provence in Canada. The DVFs, coupled with real-time location data of the workers can help in preventing accidents related to falling, collisions, etc. Two preliminary case studies show that there is a good potential for using BIM to improve safety on-site. The conclusions of the paper are: (1) A method has been proposed to automatically define areas with potential risk of falling using the 4D model of the building, (2) Then pro- 
posed method automatically generates DVFs along hazardous areas to check if the physical fences are located at the right location with the right dimension and during the right duration to prevent workers from falling, (3) The proposed method also automatically defines workspaces for each construction task, and (4) The UWB system is integrated in the proposed approach to detect the locations of the workers and to send them warnings when they approach hazardous workspaces.

More investigation and more complex case studies should be applied to further investigate the benefits of using BIM for improving safety on site. Furthermore, other safety hazards with spatial characteristics presented in Table 1 can be used to investigate BIM-based prevention program for construction safety.

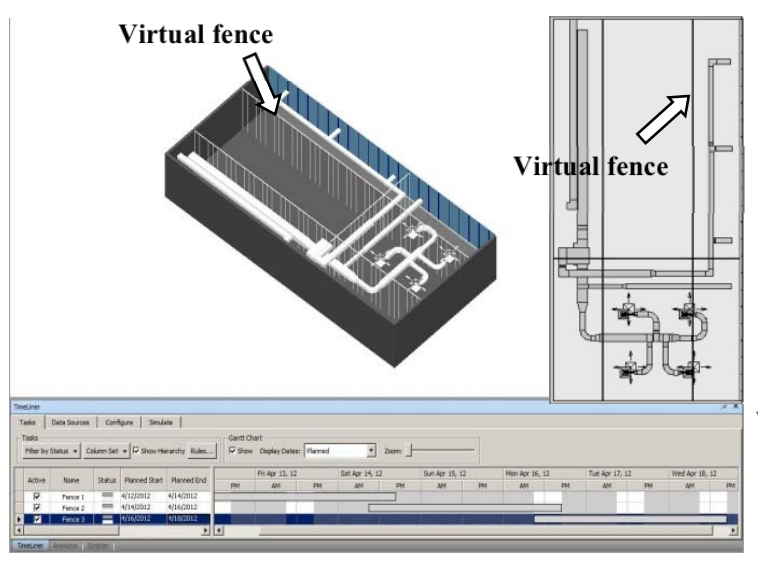

(a) BIM tool interface

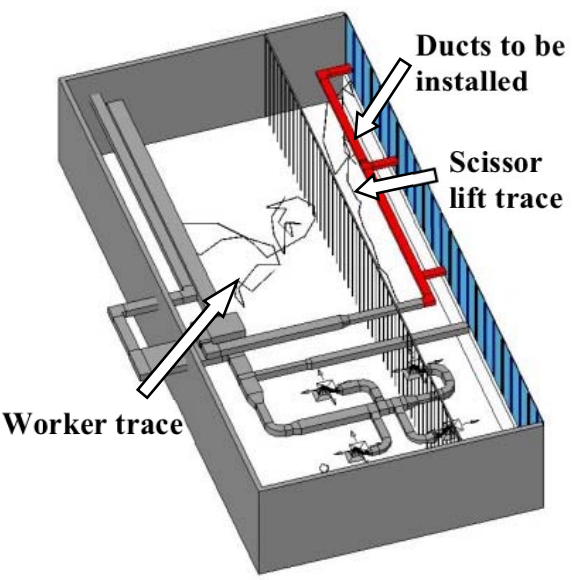

(b) Adding Traces

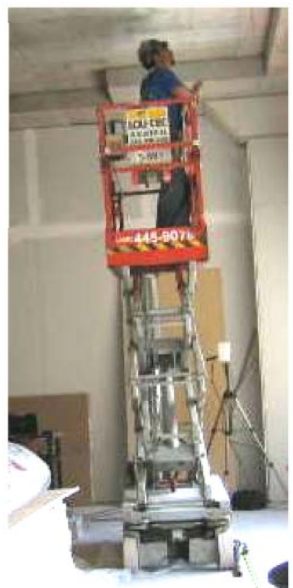

(c) Scissor lift

Figure 6: Dynamic virtual fences for HVAC ducts installation

\section{ACKNOWLEDGMENTS}

We would like to thank ASP Construction for providing the safety code materials.

\section{REFERENCES}

ASP Construction. 2003. Safety Code for the Construction industry, S-2.1, r.6.

Autodesk Navisworks. 2012. Accessed March 07, 2012. usa.autodesk.com/navisworks/.

Autodesk Revit Architecture. 2012. Accessed March 07, 2012. usa.autodesk.com/adsk/servlet/pc/index?id=17801984\&siteID=123112.

Autodesk Revit MEP. 2012. Accessed March 07, 2012. usa.autodesk.com/revit-mep/.

Eastman, C., Teicholz, P., Sacks, R. and Liston, K. 2011. BIM handbook: a guide to building information modeling for owners, managers, designers, engineers, and contractors, John Wiley and Sons.

Hammad, A., Y. Asen, and C. Zhang. 2011. "Visualizing Construction Processes using Augmented Reality: Fusing BIM, Video Monitoring and Location Information." In the 11th International Conference on Construction Applications of Virual Reality 2011, Weimar, Germany.

Huang, X., and J. Hinze. 2003. "Analysis of Construction Worker Fall Accidents." Journal of Construction Engineering and Management, ASCE, 129(3): 262-271.

Kiviniemi, M., K. Sulankivi, K. Kahkonen, T. Makele, and M.L. Merivirta. 2011. "BIM-based Safety Management and Communication for Building Construction." VTT research notes 2597. Accessed March 07, 2012. www.vtt.fi/inf/pdf/tiedotteet/2011/T2597.pdf. 
Zhang, C., A. Hammad, S. Setayeshgar, M. Soltani, A. M. 2012. "Dynamic Virtual Fences for Improving Workers Safety using BIM and RTLS." In Proceedings of the 14th International Conference on Computing in Civil and Building Engineering, Moscow, June, 2012. (paper accepted)

Zhang, S., J.K. Lee, M. Venugopal, J. Teizer, and C. Eastman. 2011. "Integrating BIM and Safety: An Automated Rule-Based Checking System for Safety Planning and Simulation." In Proceedings of CIB W099, Washington D.C., August, 2011.

\section{AUTHOR BIOGRAPHIES}

AMIN HAMMAD is a professor at the Concordia Institute for Information Systems Engineering. His research focuses on sustainable infrastructure lifecycle management systems and investigates several methods and techniques including spatio-temporal information modeling and analysis, simulation, visualization, optimization, wireless communications, sensing, auto-identification, and real-time location tracking. His email address is hammad@ciise.concordia.ca.

CHENG ZHANG is a post-doctoral fellow in the Building, Civil and Environmental Engineering Department at Concordia University in Montreal, Canada. She received her doctorate in Building Engineering from Concordia University. Her research interests are in construction engineering and management, information technology applications in construction, building information modeling and simulation, smart construction site, and sustainable construction, multi-agent system for construction. Her email address is zha_che@encs.concordia.ca.

SHAYAN SETAYESHGAR is a graduate research assistant and Master student in the Building, Civil and Environmental Engineering Department at Concordia University in Montreal, Canada. His research areas are in building information modeling, real-time location systems, and information technology applications in construction management. His email address is shayan_setayeshgar@yahoo.com.

YOOSEF ASEN is a graduate research assistant and Master student in the Building, Civil and Environmental Engineering Department at Concordia University in Montreal, Canada. His research interests are in construction engineering and management, building information modeling, and information technology applications in facilities management. His email address is y_asen@encs.concordia.ca. 